\title{
Nanoscale Investigation of Thermal Alteration of Chondritic Meteorites via Simultaneous Secondary and Transmitted Electron Imaging during In Situ Heating up to $1000{ }^{\circ} \mathrm{C}$
}

Jane Y. Howe ${ }^{1,2}$, Pierre Haenecour ${ }^{2}$, Michelle S. Thompson ${ }^{3}$, Stas Dogel $^{4}$, Takeshi Sunaoshi ${ }^{5}$, James Sagar ${ }^{6}$, Hooman Hosseinkhannazer ${ }^{7}$, and Thomas J. Zega ${ }^{2,8}$

1. Hitachi High Technologies America Inc., Clarksburg, USA.

2. Lunar and Planetary Laboratory, University of Arizona, Tucson, USA.

3 NASA Johnson Space Center, Houston, USA.

4. Hitachi High Technologies Canada Inc., Toronto, Canada.

5. Hitachi High Technologies Co., Hitachinaka, Japan.

6. Oxford Instruments NanoAnalysis, High Wycombe, UK.

7. Norcada Inc., Edmonton, Canada.

8. Materials Science and Engineering, University of Arizona, Tucson, USA.

Chondritic meteorites are among the most primitive samples available for study and provide unique insight into the processes that occurred during the early stages of solar-system formation. While they have been affected by aqueous alteration on their parent-body asteroids, the Murchison and Tagish Lake carbonaceous chondrites experienced minimal thermal alteration $[1,2]$. They contain high-levels of carbonaceous compounds and water-bearing phyllosilicates [3], and the response of these (and other) materials to external heating has important implications for the history of these meteorites and active processes occurring on their parent bodies. We are interested in learning the effects of thermal alteration on the physical and mineralogical structure of fine-grained material in carbonaceous chondrites from the micro- to the nanoscale.

Herein we report on in situ heating of the fine-grained matrix material of the Murchison meteorite in a Hitachi SU9000 STEM/SEM equipped with an Oxford X-Max ${ }^{\mathrm{N}}$ 100LE EDS system and a Hitachi Blaze heating and electric bias sample holder. Using a Norcada MEMS heating chip, we proceeded with step heating at temperature intervals of $200(16 \mathrm{~m}), 400(12 \mathrm{~m}), 600(11 \mathrm{~m}), 800(2 \mathrm{~m})$, and $1000{ }^{\circ} \mathrm{C}(10 \mathrm{~m})$. This heating schedule covered the thermal alteration range from aqueous processes, to thermal metamorphism, and to partial melting. During heating, videos were recorded at $30 \mathrm{fpm}$ to observe any potential structural changes. After reaching each step, the temperature was held isothermally at $200{ }^{\circ} \mathrm{C}$ for imaging and EDS mapping. Simultaneous secondary electron (SE) and scanning transmitted electron (bright-field, BF and annular dark field, ADF) imaging at $30 \mathrm{kV}$ reveal the changes that occur on the surface (SE) and throughout the bulk (ADF and BF).

Matrix material from the Murchison CM2 chondrite was drop-casted onto the heating chip. We surveyed the sample and performed EDS mapping of disaggregated Murchison matrix prior to heating. The EDS maps show that the local area is composed of $\mathrm{Fe}, \mathrm{Mg}, \mathrm{S}, \mathrm{Al}, \mathrm{Ni}, \mathrm{O}$, and $\mathrm{C}$, consistent with the silicate and C-bearing mixture that occurs in the matrix of the Murchison CM2 chondrite [1]. Heating at $200{ }^{\circ} \mathrm{C}$ did not bring any noticeable change to the sample in terms of grain structure, surface morphology, or elemental composition. Although heating up to $600{ }^{\circ} \mathrm{C}$ introduced little difference in the elemental composition, there is a subtle change of surface morphology. After a 2 min hold at $800{ }^{\circ} \mathrm{C}$, we observed $\mathrm{Fe}-\mathrm{Ni}$ nanoparticles formed on the surface of some grains with silicate-like compositions, along with enrichment of aluminum and carbon in certain areas (Fig. 2). These nanoparticles are similar in size to those observed in space-weathering-induced nanophase iron that form on the surface of lunar soil particles [4]. During the 10-min heating at $1000{ }^{\circ} \mathrm{C}$, extensive melting and sintering occurred, and the 
Fe-Ni nanoparticles grew in size but reduced in numbers. Comparing Fig. 2 and 3, the volume of the local area was significantly reduced in size, suggesting loss of presumably volatile components. Further, we noticed the formation of Al-rich nanocrystals (Fig. 3e) and locally concentrated carbon (Fig. 3f).

To our knowledge, in situ stepwise heating experiments of carbonaceous chondrite material has not been performed inside of a STEM. The early results here reveal the efficacy of the approach to gaining insight into thermal processing of such material. Simultaneous SE/TE imaging in conjunction with EDS mapping show that obvious thermal alteration occurred at temperatures above $600{ }^{\circ} \mathrm{C}$. Research is ongoing to systematically investigate different matrix components, such as olivine, phyllosilicates, and organic matter in the Murchison and Tagish Lake meteorites. This work will provide constraints on the effects of alteration of fine-grained matrix material in these meteorites and their thermal histories [5].

\section{References:}

[1] P Schmitt-Kopplin, et al, Proc Natl Acad Sci 107 (2010), p. 2763.

[2] P G Brown, et al, Science 290 (2000), p. 320.

[3] AE Rubin, et al, Geochim Cosmochim Acta 71 (2007), p. 2361.

[4] MS Thompson, TJ Zega, and JY Howe, Meteorit Planet Sci 52 (2017), p. 413.

[5] We gratefully acknowledge NASA (grants \#NNX12AL47G and \#NNX15AJ22G) and NSF (grants \#1531243 and \#0619599) for funding of instrumentation in the Kuiper Materials Imaging and Characterization Facility at the Lunar and Planetary Laboratory, University of Arizona. Research supported in part by NASA grant \#NNX15AJ22G.
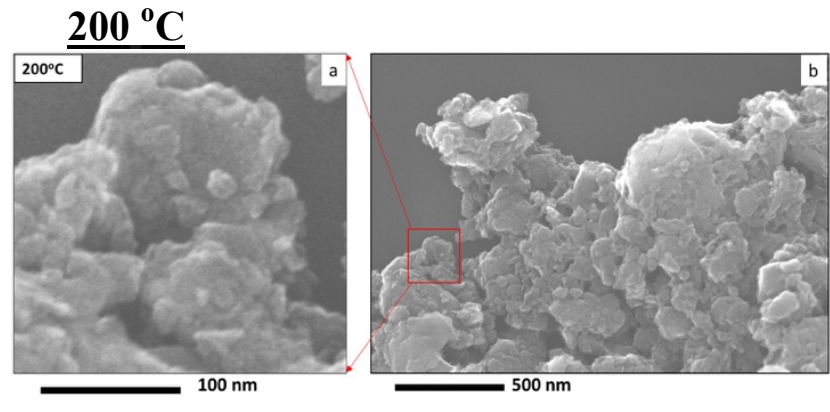

Fig. 1. SE images of Murchison meteorite after heating at $200{ }^{\circ} \mathrm{C}$.

\section{$1000{ }^{\circ} \mathrm{C}$}

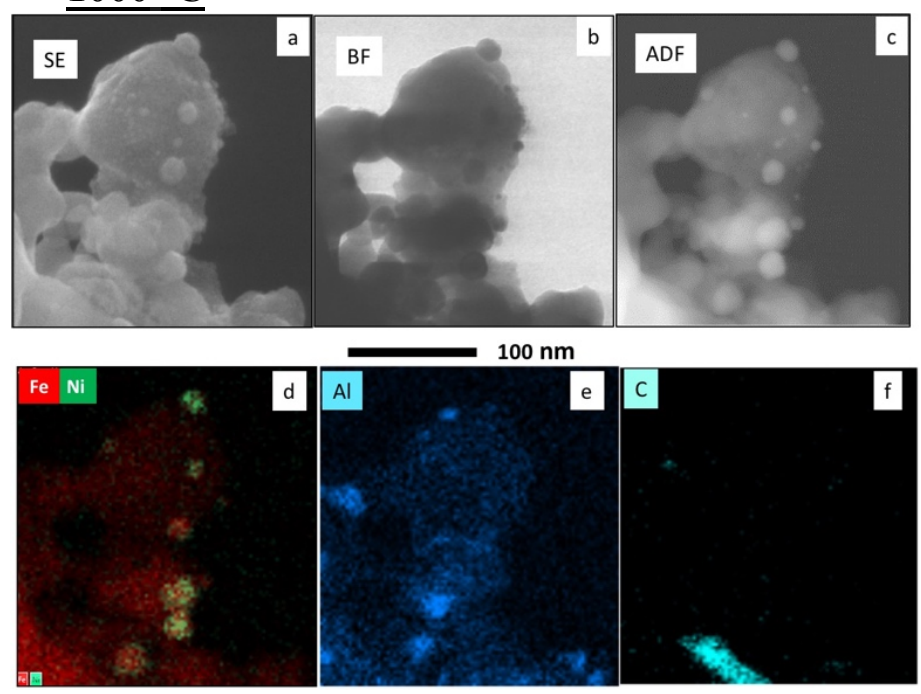

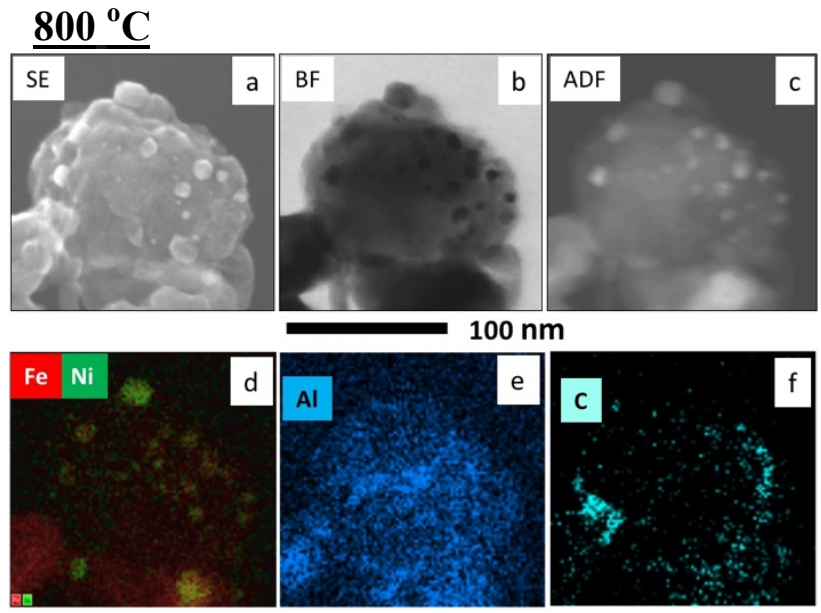

Fig. 2. Simultaneous $\mathrm{SE}, \mathrm{BF}$ and $\mathrm{ADF}$ images and EDS maps after heating at $800{ }^{\circ} \mathrm{C}$. Fe- and Ni-rich nanoparticles formed.
Fig. 3. Simultaneous $\mathrm{SE}, \mathrm{BF}$ and $\mathrm{ADF}$ images and EDS maps after heating at $1000{ }^{\circ} \mathrm{C}$. Reduced volume of the main features. 